Proyecciones Journal of Mathematics

Vol. 36, No 4, pp. 685-699, December 2017.

Universidad Católica del Norte

Antofagasta - Chile

\title{
Rough statistical convergence on triple sequences
}

\author{
Shyamal Debnath \\ Tripura University, India \\ and \\ N. Subramanian \\ Sastra University, India \\ Received : January 2017. Accepted : July 2017
}

\begin{abstract}
In this paper, using the concept of natural density, we introduce the notion of rough statistical convergence of triple sequences. We define the set of rough statistical limit points of a triple sequence and obtain rough statistical convergence criteria associated with this set. Later, we prove this set is closed and convex and also examine the relations between the set of rough statistical cluster points and the set of rough statistical limit points of a triple sequence.
\end{abstract}

Keywords : Rough statistical convergence, Natural density, triple sequences, chi sequence.

Mathematics Subject Classification 2010 : 40F05, 40J05, $40 G 05$. 


\section{Introduction}

The idea of statistical convergence was introduced by Steinhaus [12] and also independently by Fast [7] for real or complex sequences. Statistical convergence is a generalization of the usual notion of convergence, which parallels the theory of ordinary convergence.

A triple sequence (real or complex) can be defined as a function $x$ : $\mathbf{N} \times \mathbf{N} \times \mathbf{N} \rightarrow \mathbf{R}(\mathbf{C})$, where $\mathbf{N}, \mathbf{R}$ and $\mathbf{C}$ denote the set of natural numbers, real numbers and complex numbers respectively. The different types of notions of triple sequence was introduced and investigated at the initial by Sahiner et al. [10,11], Esi et al. [4-6], Datta et al. [2], Subramanian et al. [13], Debnath et al. [3] and many others.

Let $K$ be a subset of the set $\mathbf{N} \times \mathbf{N} \times \mathbf{N}$, and let us denote the set $\{(m, n, k) \in K: m \leq u, n \leq v, k \leq w\}$ by $K_{u v w}$. Then the natural density of $K$ is given by $\delta(K)=\lim _{u v w \rightarrow \infty} \frac{\left|K_{u v w}\right|}{u v w}$, where $\left|K_{u v w}\right|$ denotes the number of elements in $K_{u v w}$. Clearly, a finite subset has natural density zero, and we have $\delta\left(K^{c}\right)=1-\delta(K)$ where $K^{c}=\mathbf{N} \backslash K$ is the complement of $K$. If $K_{1} \subseteq K_{2}$, then $\delta\left(K_{1}\right) \leq \delta\left(K_{2}\right)$.

Consider a triple sequence $x=\left(x_{m n k}\right)$ such that $x_{m n k} \in \mathbf{R}, m, n, k \in \mathbf{N}$.

A triple sequence $x=\left(x_{m n k}\right)$ is said to be statistically convergent to $0 \in \mathbf{R}$, written as $s t-\lim x=0$, provided that the set

$$
\left\{(m, n, k) \in \mathbf{N}^{3}:\left|x_{m n k}-0\right| \geq \epsilon\right\}
$$

has natural density zero for any $\epsilon>0$. In this case, 0 is called the statistical limit of the triple sequence $x$.

If a triple sequence is statistically convergent, then for every $\epsilon>0$, infinitely many terms of the sequence may remain outside the $\epsilon-$ neighbourhood of the statistical limit, provided that the natural density of the set consisting of the indices of these terms is zero. This is an important property that distinguishes statistical convergence from ordinary convergence. Because the natural density of a finite set is zero, we can say that every ordinary convergent sequence is statistically convergent.

If a triple sequence $x=\left(x_{m n k}\right)$ satisfies some property $P$ for all $m, n, k$ except a set of natural density zero, then we say that the triple sequence 
$x$ satisfies $P$ for "almost all $(m, n, k)$ " and we abbreviate this by "a.a. $(m, n, k)$ ".

Let $\left(x_{m_{i} n_{j} k_{\ell}}\right)$ be a sub sequence of $x=\left(x_{m n k}\right)$. If the natural density of the set $K=\left\{\left(m_{i}, n_{j}, k_{\ell}\right) \in \mathbf{N}^{3}:(i, j, \ell) \in \mathbf{N}^{3}\right\}$ is different from zero, then $\left(x_{m_{i} n_{j} k_{\ell}}\right)$ is called a non thin sub sequence of a triple sequence $x$.

$c \in \mathbf{R}$ is called a statistical cluster point of a triple sequence $x=\left(x_{m n k}\right)$ provided that the natural density of the set

$$
\left\{(m, n, k) \in \mathbf{N}^{3}:\left|x_{m n k}-c\right|<\epsilon\right\}
$$

is different from zero for every $\epsilon>0$. We denote the set of all statistical cluster points of the sequence $x$ by $\Gamma_{x}$.

A triple sequence $x=\left(x_{m n k}\right)$ is said to be statistically analytic if there exists a positive number $M$ such that

$$
\delta\left(\left\{(m, n, k) \in \mathbf{N}^{3}:\left|x_{m n k}\right|^{1 / m+n+k} \geq M\right\}\right)=0
$$

The theory of statistical convergence has been discussed in trigonometric series, summability theory, measure theory, turnpike theory, approximation theory, fuzzy set theory and so on.

The idea of rough convergence was introduced by Phu [9], who also introduced the concepts of rough limit points and roughness degree. The idea of rough convergence occurs very naturally in numerical analysis and has interesting applications. Aytar [1] extended the idea of rough convergence into rough statistical convergence using the notion of natural density just as usual convergence was extended to statistical convergence. Pal et al. [8] extended the notion of rough convergence using the concept of ideals which automatically extends the earlier notions of rough convergence and rough statistical convergence.

In this paper, we introduce the notion of rough statistical convergence of triple sequences. Defining the set of rough statistical limit points of a triple sequence, we obtain rough statistical convergence criteria associated with this set.

Throughout the paper $r$ be a nonnegative real number. 


\section{Definitions and Preliminaries}

\subsection{Definition}

A triple sequence $x=\left(x_{m n k}\right)$ is said to be rough convergent $(r-$ convergent $)$ to $l$ (Pringsheim's sense), denoted as $x_{m n k} \rightarrow^{r} l$, provided that

$$
\forall \epsilon>0, \exists i_{\epsilon} \in \mathbf{N}: m, n, k \geq i_{\epsilon} \Longrightarrow\left|x_{m n k}-l\right|<r+\epsilon,
$$

or equivalently, if

$$
\lim \sup \left|x_{m n k}-l\right| \leq r .
$$

Here $r$ is called the roughness of degree. If we take $r=0$, then we obtain the ordinary convergence of a triple sequence.

\subsection{Definition}

It is obvious that the $r$ - limit set of a triple sequence is not unique. The $r$ - limit set of the triple sequence $x=\left(x_{m n k}\right)$ is defined as $L I M^{r} x_{m n k}:=$ $\left\{l \in \mathbf{R}: x_{m n k} \rightarrow^{r} l\right\}$.

\subsection{Definition}

A triple sequence $x=\left(x_{m n k}\right)$ is said to be $r$ - convergent if $L I M^{r} x \neq \phi$. In this case, $r$ is called the convergence degree of the triple sequence $x=$ $\left(x_{m n k}\right)$. For $r=0$, we get the ordinary convergence.

\subsection{Definition}

A triple sequence $\left(x_{m n k}\right)$ is said to be $r$ - statistically convergent to $l$, denoted by $x_{m n k} \rightarrow^{r s t} l$, provided that the set

$$
\left\{(m, n, k) \in \mathbf{N}^{3}:\left|x_{m n k}-l\right| \geq r+\epsilon\right\}
$$

has natural density zero for every $\epsilon>0$, or equivalently, if the condition

$$
\text { st - lim sup }\left|x_{m n k}-l\right| \leq r
$$

is satisfied.

In addition, we can write $x_{m n k} \rightarrow^{r s t} l$ if and only if the inequality

$$
\left|x_{m n k}-l\right|<r+\epsilon
$$


holds for every $\epsilon>0$ and almost all $(m, n, k)$. Here $r$ is called the roughness of degree. If we take $r=0$, then we obtain the statistical convergence of triple sequences.

In a similar fashion to the idea of classical rough convergence, the idea of rough statistical convergence of a triple sequence can be interpreted as follows:

Assume that a triple sequence $y=\left(y_{m n k}\right)$ is statistically convergent and cannot be measured or calculated exactly; one has to do with an approximated (or statistically approximated) triple sequence $x=\left(x_{m n k}\right)$ satisfying $\left|x_{m n k}-y_{m n k}\right| \leq r$ for all $m, n, k$ (or for almost all $(m, n, k)$, i.e.,

$$
\delta\left(\left\{(m, n, k) \in \mathbf{N}^{3}:\left|x_{m n k}-y_{m n k}\right|>r\right\}\right)=0 .
$$

Then the triple sequence $x$ is not statistically convergent any more, but as the incluson

$$
\left\{(m, n, k) \in \mathbf{N}^{3}:\left|y_{m n k}-l\right| \geq \epsilon\right\} \supseteq\left\{(m, n, k) \in \mathbf{N}^{3}:\left|x_{m n k}-l\right| \geq r+\epsilon\right\}
$$

holds and we have

$$
\delta\left(\left\{(m, n, k) \in \mathbf{N}^{3}:\left|y_{m n k}-l\right| \geq \epsilon\right\}\right)=0,
$$

i.e., we get

$$
\delta\left(\left\{(m, n, k) \in \mathbf{N}^{3}:\left|x_{m n k}-l\right| \geq r+\epsilon\right\}\right)=0,
$$

i.e., the triple sequence spaces $x$ is $r$ - statistically convergent in the sense of definition (2.3)

In general, the rough statistical limit of a triple sequence may not unique for the roughness degree $r>0$. So we have to consider the so called $r-$ statistical limit set of a triple sequence $x=\left(x_{m n k}\right)$, which is defined by

$$
\text { st }-L I M^{r} x=\left\{L \in \mathbf{R}: x_{m n k} \rightarrow^{r s t} l\right\} .
$$

The triple sequence $x$ is said to be $r$ - statistically convergent provided that $s t-L I M^{r} x \neq \phi$. It is clear that if $s t-L I M^{r} x \neq \phi$. for a triple sequence $x=\left(x_{m n k}\right)$ of real numbers, then we have

$$
s t-L I M^{r} x=[s t-\lim \sup x-r, s t-\lim \inf x+r]
$$

We know that $L I M^{r} x=\phi$ for an unbounded triple sequence $x=\left(x_{m n k}\right)$. But such a triple sequence might be rough statistically convergent. For instance, define 


$$
x_{m n k}=\left\{\begin{array}{cc}
(-1)^{m n k}, & \text { if }(m, n, k) \neq(i, j, \ell)^{2}(i, j, \ell \in \mathbf{N}), \\
(m n k), & \text { otherwise }
\end{array}\right\} .
$$

in $\mathbf{R}$. Because the set $\{1,64,739, \cdots\}$ has natural density zero, we have

$$
s t-L I M^{r} x=\left\{\begin{array}{cc}
\phi, & \text { if } r<1, \\
{[1-r, r-1],} & \text { otherwise }
\end{array}\right\}
$$

and $L I M^{r} x=\phi$ for all $r \geq 0$.

As can be seen by the example above, the fact that $s t-L I M^{r} x \neq \phi$ does not imply $L I M^{r} x \neq \phi$. Because a finite set of natural numbers has natural density zero, $L I M^{r} x \neq \phi$ implies $s t-L I M^{r} x \neq \phi$. Therefore, we get $L I M^{r} x \subseteq s t-L I M^{r} x$. This obvious fact means $\left\{r \geq 0: L I M^{r} x \neq \phi\right\} \subseteq$ $\left\{r \geq 0: s t-L I M^{r} x \neq \phi\right\}$ in this language of sets and yields immediately

$$
\text { inf }\left\{r \geq 0: L I M^{r} x \neq \phi\right\} \geq \inf \left\{r \geq 0: s t-L I M^{r} x \neq \phi\right\} .
$$

Moreover, it also yields directly $\operatorname{diam}\left(L I M^{r} x\right) \leq \operatorname{diam}\left(s t-L I M^{r} x\right)$.

\section{Main Results}

\subsection{Theorem}

For a triple sequence spaces $x=\left(x_{m n k}\right)$, we have $\operatorname{diam}\left(s t-L I M^{r} x\right) \leq 2 r$. In general diam $\left(s t-L I M^{r} x\right)$ has an upper bound.

Proof: Assume that $\operatorname{diam}\left(s t-L I M^{r} x\right)>2 r$. Then there exist $w, y \in$ st $-L I M^{r} x$ such that $|w-y|>2 r$. Take $\epsilon \in\left(0, \frac{|w-y|}{2}-r\right)$. Because $w, y \in$ st $-L I M^{r} x$, we have $\delta\left(K_{1}\right)=0$ and $\delta\left(K_{2}\right)=0$ for every $\epsilon>0$ where

$$
K_{1}=\left\{(m, n, k) \in \mathbf{N}^{3}:\left|x_{m n k}-w\right| \geq r+\epsilon\right\}
$$

and

$$
K_{2}=\left\{(m, n, k) \in \mathbf{N}^{3}:\left|x_{m n k}-y\right| \geq r+\epsilon\right\} .
$$

Using the properties of natural density, we get $\delta\left(K_{1}^{c} \cap K_{2}^{c}\right)=1$. Thus we can write

$|w-y| \leq\left|x_{m n k}-w\right|+\left|x_{m n k}-y\right|$ 


$$
<2(r+\epsilon)=2\left(\frac{|w-y|}{2}\right)=|w-y|
$$

for all $(m, n, k) \in K_{1}^{c} \cap K_{2}^{c}$, which is a contradiction.

Now let us prove the second part of the theorem. Consider a triple sequence $x=\left(x_{m n k}\right)$ such that $s t-\lim x_{m n k}=l$. Let $\epsilon>0$. Then we can write

$$
\begin{aligned}
& \delta\left(\left\{(m, n, k) \in \mathbf{N}^{3}:\left|x_{m n k}-l\right| \geq \epsilon\right\}\right)=0 . \text { We have } \\
& \qquad\left|x_{m n k}-y\right| \leq\left|x_{m n k}-l\right|+|l-y| \leq\left|x_{m n k}-l\right|+r
\end{aligned}
$$

for each $y \in \bar{B}_{r}(l)=\left\{y \in \mathbf{R}^{3}:|y-l| \leq r\right\}$.

Then we get $|l-y|<r+\epsilon$ for each $(m, n, k) \in\left\{(m, n, k) \in \mathbf{N}^{3}:\left|x_{m n k}-l\right|<\epsilon\right\}$. Because the triple sequence spaces $x$ is statistically convergent to $l$, we have

$$
\delta\left(\left\{(m, n, k) \in \mathbf{N}^{3}:\left|x_{m n k}-l\right|<\epsilon\right\}\right)=1 .
$$

Therefore we get $y \in s t-L I M^{r} x$. Hence, we can write

$$
s t-L I M^{r} x=\bar{B}_{r}(l) .
$$

Because $\operatorname{diam}\left(\bar{B}_{r}(l)\right)=2 r$, this shows that in general, the upper bound $2 r$ of the diameter of the set $s t-L I M^{r} x$ is not an lower bound.

\subsection{Theorem}

Let $r>0$. Then a triple sequence $x=\left(x_{m n k}\right)$ is $r$ - statistically convergent to $l$ if and only if there exists a triple sequence $y=\left(y_{m n k}\right)$ such that $s t-\lim y=l$ and $\left|x_{m n k}-y_{m n k}\right| \leq r$ for each $(m, n, k) \in \mathbf{N}^{3}$.

Proof: Necessity: Assume that $x_{m n k} \rightarrow^{r s t} l$. Then we have

$$
\text { st }-\lim \sup \left|x_{m n k}-l\right| \leq r .
$$

Now, define

$$
\begin{gathered}
y_{m n k}=\left\{l, \text { if }\left|x_{m n k}-l\right| \leq r,\right. \\
\mathrm{x}_{m n k}+r\left(\frac{l-x_{m n k}}{\left|x_{m n k}-l\right|}\right), \text { otherwise, }
\end{gathered}
$$

Then, we write

$$
\begin{gathered}
\left|y_{m n k}-l\right|=\left\{\quad \mid l-l, \text { if }\left|x_{m n k}-l\right| \leq r\right. \\
\left|x_{m n k}-l\right|+r\left(\frac{|l-l|-\left|x_{m n k}-l\right|}{\left|x_{m n k}-l\right|}\right), \text { otherwise, }
\end{gathered}
$$


(i.e) $\left|y_{m n k}-l\right|=\left\{0\right.$, if $\left|x_{m n k}-l\right| \leq r$,

$\left|x_{m n k}-l\right|-r\left(\frac{\left|x_{m n k}-l\right|}{\left|x_{m n k}-l\right|}\right)$, otherwise,

(i.e) $\left|y_{m n k}-l\right|=\left\{0\right.$, if $\left|x_{m n k}-l\right| \leq r$,

$\left|x_{m n k}-l\right|-r$, otherwise.

We have $\left|y_{m n k}-l\right| \geq\left|x_{m n k}-l\right|-r \Longrightarrow\left|x_{m n k}-l-y_{m n k}+l\right| \leq r$

$$
\left|x_{m n k}-y_{m n k}\right| \leq r
$$

for all $m, n, k \in \mathbf{N}$. By equation (3.1) and by definition of $y_{m n k}$, we get st - limsup $\left|y_{m n k}-l\right|=0$.

$$
\Longrightarrow s t-\lim y_{m n k} \rightarrow^{r} l \text {. }
$$

Sufficiency: Because $s t-\lim y_{m n k}=l$, we have

$$
\delta\left(\left\{(m, n, k) \in \mathbf{N}^{3}:\left|y_{m n k}-l\right| \geq \epsilon\right\}\right)=0
$$

for each $\epsilon>0$. It is easy to see that the inclusion

$$
\left\{(m, n, k) \in \mathbf{N}^{3}:\left|y_{m n k}-l\right| \geq \epsilon\right\} \supseteq\left\{(m, n, k) \in \mathbf{N}^{3}:\left|x_{m n k}-l\right| \geq r+\epsilon\right\}
$$

holds. Because $\delta\left(\left\{(m, n, k) \in \mathbf{N}^{3}:\left|y_{m n k}-l\right| \geq \epsilon\right\}\right)=0$, we get $\delta\left(\left\{(m, n, k) \in \mathbf{N}^{3}:\left|x_{m n k}-l\right| \geq r+\epsilon\right\}\right)=0$.

\subsection{Remark}

If we replace the condition $\left|x_{m n k}-y_{m n k}\right| \leq r$ for all $m, n, k \in \mathbf{N}$ in the hypothesis of the Theorem (3.2) with the condition

$$
\delta\left(\left\{(m, n, k) \in \mathbf{N}^{3}:\left|x_{m n k}-y_{m n k}\right|>r\right\}\right)=0
$$

is valid.

\subsection{Theorem}

For an arbitrary $c \in \Gamma_{x}$ of triple sequence $x=\left(x_{m n k}\right)$ we have $|l-c| \leq r$ for all $l \in s t-L I M^{r} x$.

Proof: Assume on the contrary that there exist a point $c \in \Gamma_{x}$ and $l \in$ $s t-L I M^{r} x$ such that $|l-c|>r$. Define $\epsilon:=\frac{|l-c|-r}{3}$. Then

$$
\left(3 .\left\{Y(m, n, k) \in \mathbf{N}^{3}:|l-c|<\epsilon\right\} \subseteq\left\{(m, n, k) \in \mathbf{N}^{3}:\left|x_{m n k}-l\right| \geq r+\epsilon\right\} .\right.
$$

Since $c \in \Gamma_{x}$, we have 


$$
\delta\left(\left\{(m, n, k) \in \mathbf{N}^{3}:\left|x_{m n k}-c\right|<\epsilon\right\}\right) \neq 0 .
$$

Hence, by (3.3), we get

$$
\delta\left(\left\{(m, n, k) \in \mathbf{N}^{3}:\left|x_{m n k}-l\right| \geq r+\epsilon\right\}\right) \neq 0,
$$

which contradicts the fact $l \in s t-L I M^{r} x$.

\subsection{Proposition}

If a triple sequence $x=\left(x_{m n k}\right)$ is analytic, then there exists a non-negative real number $r$ such that $s t-L I M^{r} x \neq \phi$.

Proof: If we take the triple sequence is to be statistically analytic, then the of proposition holds. Thus we have the following theorem.

\subsection{Theorem}

A triple sequence $x=\left(x_{m n k}\right)$ is statistically analytic if and only if there exists a non-negative real number $r$ such that $s t-L I M^{r} x \neq \phi$.

Proof: Since the triple sequence $x$ is statistically analytic, there exists a positive real number $M$ such that

$$
\delta\left(\left\{(m, n, k) \in \mathbf{N}^{3}:\left|x_{m n k}\right|^{1 / m+n+k} \geq M\right\}\right)=0 .
$$

Define

$$
r^{\prime}=\sup \left\{\left|x_{m n k}\right|^{1 / m+n+k}:(m, n, k) \in K^{c}\right\}
$$

where

$$
K=\left\{(m, n, k) \in \mathbf{N}^{3}:\left|x_{m n k}\right|^{1 / m+n+k} \geq M\right\} .
$$

Then the set $s t-L I M^{r}{ }^{\prime} x$ contains the origin of $\mathbf{R}$. So we have $s t-$ $L I M^{r} x \neq \phi$.

If $s t-L I M^{r} x \neq \phi$ for some $r \geq 0$, then there exists $l$ such that $l \in s t-L I M^{r} x$, i.e.,

$$
\delta\left(\left\{(m, n, k) \in \mathbf{N}^{3}:\left|x_{m n k}-l\right|^{1 / m+n+k} \geq r+\epsilon\right\}\right)=0 .
$$

for each $\epsilon>0$. Then we say that almost all $x_{m n k}$ are contained in some ball with any radius greater than $r$. So the triple sequence $x$ is statistically analytic. 


\subsection{Remark}

If $x^{\prime}=\left(x_{m_{i}, n_{j}, k_{\ell}}\right)$ is a sub sequence of $x=\left(x_{m n k}\right)$, then $L I M^{r} x \subseteq$ $L I M^{r} x^{\prime}$. But it is not valid for statistical convergence. For example, define

$$
x_{m n k}=\left\{\begin{array}{cc}
(m n k), & \text { if }(m, n, k)=(i, j, \ell)^{2}(i, j, \ell \in \mathbf{N}), \\
0, & \text { otherwise }
\end{array}\right\}
$$

of real numbers. Then the triple sequence $x^{\prime}=(1,64,739, \cdots)$ is a subsequence of $x$. We have $s t-L I M^{r} x=[-r, r]$ abd $s t-L I M^{r} x^{\prime}=\phi$.

\subsection{Theorem}

Let $x^{\prime}=\left(x_{m_{i}, n_{j}, k_{\ell}}\right)$ is a non-thin subsequence of triple sequence $x=$ $\left(x_{m n k}\right)$, then $s t-L I M^{r} x \subseteq s t-L I M^{r} x^{\prime}$.

Proof: Easy, so omitted.

\subsection{Theorem}

The $r$ - statistical limit set of a triple sequence $x=\left(x_{m n k}\right)$ is closed.

Proof: If $s t-L I M^{r} x \neq \phi$, then it is true. Assume that $s t-L I M^{r} x \neq \phi$, then we can choose a triple sequence spaces $\left(y_{m n k}\right) \subseteq s t-L I M^{r} x$ such that $y_{m n k} \rightarrow^{r} l$ as $m, n, k \rightarrow \infty$. If we prove that $l \in s t-L I M^{r} x$, then the proof will be complete.

Let $\epsilon>0$ be given. Because $y_{m n k} \rightarrow^{r} l, \forall \epsilon>0, \exists i_{\epsilon} \in \mathbf{N}: m, n, k \geq i_{\epsilon}$ such that

$$
\left|y_{m n k}-l\right|<\frac{\epsilon}{2} \text { for all } \mathrm{m}, \mathrm{n}, \mathrm{k} \geq i_{\epsilon}
$$

Now choose an $\left(m_{0}, n_{0}, k_{0}\right) \in \mathbf{N}$ such that $m_{0}, n_{0}, k_{0} \geq i_{\epsilon}$. Then we can write

$$
\left|y_{m_{0} n_{0} k_{0}}-l\right|<\frac{\epsilon}{2} .
$$

On the other hand, because $\left(y_{m n k}\right) \subseteq s t-L I M^{r} x$, we have $y_{m_{0} n_{0} k_{0}} \in$ $s t-L I M^{r} x$, namely,

$$
\delta\left(\left\{(m, n, k) \in \mathbf{N}^{3}:\left|x_{m n k}-y_{m_{0} n_{0} k_{0}}\right| \geq r+\frac{\epsilon}{2}\right\}\right)=0 .
$$


Now let us show that the inclusion

$$
\begin{gathered}
\left\{(m, n, k) \in \mathbf{N}^{3}:\left|x_{m n k}-l\right|<r+\epsilon\right\} \\
\supseteq\left\{(m, n, k) \in \mathbf{N}^{3}:\left|x_{m n k}-y_{m_{0} n_{0} k_{0}}\right|<r+\frac{\epsilon}{2}\right\}
\end{gathered}
$$

holds. Take $(i, j, \ell) \in\left\{(m, n, k) \in \mathbf{N}^{3}:\left|x_{m n k}-y_{m_{0} n_{0} k_{0}}\right|<r+\frac{\epsilon}{2}\right\}$. Then we have

$$
\left|x_{m n k}-y_{m_{0} n_{0} k_{0}}\right|<r+\frac{\epsilon}{2}
$$

and hence

$$
\left|x_{i j \ell}-l\right| \leq\left|x_{i j \ell}-y_{m_{0} n_{0} k_{0}}\right|+\left|y_{m_{0} n_{0} k_{0}}-l\right|<r+\frac{\epsilon}{2}+\frac{\epsilon}{2}<r+\epsilon
$$

i.e., $(i, j, \ell) \in\left\{(m, n, k) \in \mathbf{N}^{3}:\left|x_{m n k}-l\right|<r+\epsilon\right\}$ which proves the equation (3.5). Hence the natural density of the set on the LHS of equation (3.5) is equal to 1 . So we get $\delta\left(\left\{(m, n, k) \in \mathbf{N}^{3}:\left|x_{m n k}-l\right| \geq r+\epsilon\right\}\right)=0$.

\subsection{Theorem}

The $r$ - statistical limit set of a triple sequence is convex.

Proof: Let $y_{1}, y_{2} \in s t-L I M^{r} x$ for the triple sequence $x=\left(x_{m n k}\right)$ and let $\epsilon>0$ be given. Define

$K_{1}=\left\{(m, n, k) \in \mathbf{N}^{3}:\left|x_{m n k}-y_{1}\right| \geq r+\epsilon\right\}$ and

$K_{2}=\left\{(m, n, k) \in \mathbf{N}^{3}:\left|x_{m n k}-y_{2}\right| \geq r+\epsilon\right\}$. Because $y_{1}, y_{2} \in s t-L I M^{r} x$, we have $\delta\left(K_{1}\right)=\delta\left(K_{2}\right)=0$. Thus we have

$\left|x_{m n k}-\left[(1-\lambda) y_{1}+\lambda y_{2}\right]\right|=\left|(1-\lambda)\left(x_{m n k}-y_{1}\right)+\lambda\left(x_{m n k}-y_{2}\right)\right|<r+\epsilon$, for each $(m, n, k) \in\left(K_{1}^{c} \cap K_{2}^{c}\right)$ and each $\lambda \in[0,1]$. Because $\delta\left(K_{1}^{c} \cap K_{2}^{c}\right)=$ 1 , we get

$$
\delta\left(\left\{(m, n, k) \in \mathbf{N}^{3}:\left|x_{m n k}-\left[(1-\lambda) y_{1}+\lambda y_{2}\right]\right| \geq r+\epsilon\right\}\right)=0,
$$

i.e., $\left[(1-\lambda) y_{1}+\lambda y_{2}\right] \in s t-L I M^{r} x$, which proves the convexity of the set st $-L I M^{r} x$. 


\subsection{Theorem}

A triple sequence $x=\left(x_{m n k}\right)$ statistically converges to $l$ if and only if $s t-L I M^{r} x=\bar{B}_{r}(l)$.

Proof: For the necessity part of this theorem is in proof of the Theorem (3.1).

Sufficiency: Because $s t-L I M^{r} x=\bar{B}_{r}(l) \neq \phi$, then by Theorem (3.5) we can say that the triple sequence spaces $x$ is statistically analytic. Assume on the contrary that the triple sequence spaces $x$ has another statistical cluster point $l^{\prime}$ different from $l$. Then the point

$$
\bar{l}=l+\frac{r}{\left|l-l^{\prime}\right|}\left(l-l^{\prime}\right)
$$

satisfies

$$
\begin{gathered}
\bar{l}-l^{\prime}=l-l^{\prime}+\frac{r}{\left|l-l^{\prime}\right|}\left(l-l^{\prime}\right) \\
\left|\bar{l}-l^{\prime}\right|=\left|l-l^{\prime}\right|+\frac{r}{\left|l-l^{\prime}\right|}\left(l-l^{\prime}\right) \\
\left|\bar{l}-l^{\prime}\right|=\left|l-l^{\prime}\right|+r>r .
\end{gathered}
$$

Because $l^{\prime}$ is a statistical cluster point of the triple sequence spaces $x$, by Theorem (2.4) this inequality implies that $\bar{l} \notin s t-L I M^{r} x$. This contradicts the fact $|\bar{l}-l|=r$ and $s t-L I M^{r} x=\bar{B}_{r}(l)$. Therefore, $l$ is the unique statistical cluster point of the triple sequence spaces $x$. Hence the statistical cluster point of a statistically analytic triple sequence spaces is unique, then the triple sequence spaces $x$ is statistically convergent to $l$.

\subsection{Theorem}

(a) If $c \in \Gamma_{x}$ then

$$
s t-L I M^{r} x \subseteq \bar{B}_{r}(c)
$$

$$
s t-L I M^{r} x=\bigcap_{c \in \Gamma_{x}} \bar{B}_{r}(c)=\left\{l \in \mathbf{R}^{3}: \Gamma_{x} \subseteq \bar{B}_{r}(l)\right\}
$$

Proof: (a) Assume that $l \in s t-L I M^{r} x$ and $c \in \Gamma_{x}$. Then by Theorem 3.4 , we have 


$$
|l-c| \leq r
$$

other wise we get

$$
\delta\left(\left\{(m, n, k) \in \mathbf{N}^{3}:\left|x_{m n k}-l\right| \geq r+\epsilon\right\}\right) \neq 0
$$

for $\epsilon=\frac{|l-c|-r}{3}$. This contradicts the fact $l \in s t-L I M^{r} x$.

(b) By the equation (3.9), we can write

$$
s t-L I M^{r} x \subseteq \bigcap_{c \in \Gamma_{x}} \bar{B}_{r}(c) .
$$

Now assume that $y \in \bigcap_{c \in \Gamma_{x}} \bar{B}_{r}(c)$. Then we have

$$
|y-c| \leq r
$$

for all $c \in \Gamma_{x}$, which is equivalent to $\Gamma_{x} \subseteq \bar{B}_{r}(y)$, i.e.,

$$
\bigcap_{c \in \Gamma_{x}} \bar{B}_{r}(c) \subseteq\left\{l \in \mathbf{R}: \Gamma_{x} \subseteq \bar{B}_{r}(l)\right\} .
$$

Now let $y \notin s t-L I M^{r} x$. Then there exists an $\epsilon>0$ such that

$$
\delta\left(\left\{(m, n, k) \in \mathbf{N}^{3}:|l-y| \geq r+\epsilon\right\}\right) \neq 0
$$

the existence of a statistical cluster point $c$ of the triple sequence spaces $x$ with $|y-c| \geq r+\epsilon$, i.e., $\Gamma_{x} \bar{B}_{r}(y)$ and $y \notin\left\{l \in \mathbf{R}: \Gamma_{x} \subseteq \bar{B}_{r}(l)\right\}$.

Hence $y \in s t-L I M^{r} x$ follows from $y \in\left\{l \in \mathbf{R}: \Gamma_{x} \subseteq \bar{B}_{r}(l)\right\}$, i.e.,

$$
\left\{l \in \mathbf{R}: \Gamma_{x} \subseteq \bar{B}_{r}(l)\right\} \subseteq s t-L I M^{r} x .
$$

Therefore the inclusions (3.11)-(3.13) ensure that (3.10) holds.

Competing Interests: The authors declare that there is not any conflict of interests regarding the publication of this manuscript.

\section{Acknowledgements}

The present paper was completed during a visit by Professor N. Subramanian to Tripura (A central) University (May-June,2016). The research was supported by INSA (Indian National Science Academy visiting fellowship) 
while the second author was visiting Tripura (A central) University under the INSA visiting fellowship and also the authors are extremely grateful to the anonymous learned referee(s) for their keen reading, valuable suggestion and constructive comments for the improvement of the manuscript. The authors are thankful to the editor(s) and reviewers of Proyecciones Journal of Mathematics.

\section{References}

[1] S. Aytar Rough statistical Convergence, Numer. Funct. Anal. Optimi., 29 (3), pp. 291-303, (2008).

[2] A. J. Datta A. Esi and B. C. Tripathy, Statistically convergent triple sequence spaces defined by Orlicz function, J Math. Anal., 4 (2), pp. 16-22, (2013).

[3] S. Debnath, B. Sarma and B. C. Das ,Some generalized triple sequence spaces of real numbers, J. Nonlinear Anal. Optimi., 6(1), (2015), 7179 .

[4] A. Esi, On some triple almost lacunary sequence spaces defined by Orlicz functions, Research and Reviews: Discrete Mathematical Structures, 1 (2), pp. 16-25, (2014).

[5] A. Esi and M. Necdet Catalbas, Almost convergence of triple sequences, Global J. Math. Anal., 2 (1), pp. 6-10, (2014).

[6] A. Esi and E. Savas, On lacunary statistically convergent triple sequences in probabilistic normed space, Appl. Math. Inf. Sci., 9 (5), pp. 2529-2534, (2015).

[7] H. Fast, Sur la convergence statistique, Colloq. Math. 2, pp. 241-244, (1951).

[8] S. K. Pal, D. Chandra and S. Dutta Rough ideal Convergence, Hacee. J. Math. and Stat., 42 (6), pp. 633-640, (2013).

[9] H. X. Phu Rough convergence in normed linear spaces, Numer. Funct. Anal. Optimi., 22, pp. 201-224, (2001). 
[10] A. Sahiner, M. Gurdal and F. K. Duden, Triple sequences and their statistical convergence, Selcuk J. Appl. Math. , 8 (2), pp. 49-55, (2007).

[11] A. Sahiner, B. C. Tripathy, Some I related properties of triple sequences, Selcuk J. Appl. Math. , 9 (2), pp. 9-18, (2008).

[12] H.Steinhaus Sur la convergence ordinaire et la convergence asymptotique, Colloq. Math. 2, pp. 73-74, (1951).

[13] N. Subramanian and A. Esi, The generalized tripled difference of $\chi^{3}$ sequence spaces, Global Journal of Mathematical Analysis, 3 (2), pp. 54-60, (2015).

\section{Shyamal Debnath}

Department of Mathematics, Tripura University (A Central University), Suryamaninagar, Agartala-799022, West Tripura, India

e-mail : shyamalnitamath@gmail.com

and

\section{N. Subramanian}

Department of Mathematics, SASTRA University, Thanjavur-613 401, India

e-mail : nsmaths@yahoo.com 